\title{
Comparison of Tropane Alkaloid Spectra Between Datura innoxia Grown in Egypt and Bulgaria
}

Strahil Berkov ${ }^{\mathrm{a}, *}$ and Rawia Zayed $^{\mathrm{b}}$

${ }^{a}$ Department of Applied Botany, Institute of Botany, Bulgarian Academy of Sciences, 23 Acad. G. Bonchev Str., 1113 Sofia, Bulgaria. E-mail: berkov@iph.bio.bas.bg

b Department of Pharmacognosy, Faculty of Pharmacy, Zagazig University, 44519 Zagazig, Egypt

* Author for correspondence and reprint requests

Z. Naturforsch. 59c, 184-186 (2004); received August 1, 2003

The alkaloid spectra of Datura innoxia plants grown in Egypt and Bulgaria were investigated by GC-MS. Thirty-eight alkaloids were detected in the roots, leaves and fruits of the plants. Five new alkaloids for D. innoxia are reported. Alkaloid spectra of Egyptian and Bulgarian plants differ significantly in respect to their alkaloid composition and main alkaloids accumulated in the plant organs.

Key words: GC-MS, Datura innoxia, Tropane Alkaloids

\section{Introduction}

Datura innoxia Mill. (Solanaceae) is an alkaloid rich species for which more than 50 tropane alkaloids have been reported (Witte et al., 1987; Lounasmaa and Tamminen, 1993; Ionkova et al., 1994). These alkaloids are used in the chemotaxonomy of Solanaceae family (Griffin and Lin, 2000). However, the biosynthesis of a particular alkaloid depends on various factors such as genetic factors, stage of development, environment. To our knowledge, there is no information on the effect of environment on the alkaloid spectra in plants of genus Datura, analysed by a sensitive method such as GC-MS. Such kind of data would contribute to the better knowledge on distribution and variability of the alkaloids within a single species on the one hand and to estimate their chemotaxonomic significance on the other hand.

As a part of our studies on the distribution of alkaloids in the genus Datura, we report on the alkaloid spectra of different plant organs from D. innoxia plants cultivated in Egypt (with tropical climate) and Bulgaria (with continental climate).

\section{Results and Discussion}

Thirty-eight alkaloids from the extracts of $D$. innoxia were identified by GC-MS (Table I). Alkaloids 9, 11, 16, 17 and 29 and 30 appear as double peaks in the GC chromatograms and show identical mass spectra. They are isomeric tropine and pseudotropine esters (Witte et al., 1987). To our knowledge, alkaloids 8, 14, 31 and 32 as well as one of the both isomers $\mathbf{2 9}$ or $\mathbf{3 0}$ are reported for the first time for $D$. innoxia.

No referent mass spectrum for alkaloid $\mathbf{1 4}$ was found in the literature or database. The compound has $\mathrm{M}^{+}$at $m / z, 241$ and base ion at $m / z 94$ indicating C-3 and C-6 substitution. An ion at $m / z, 140$ is characteristic for a hydroxyl group at C-6 whereas an ion at $m / z, 197\left[\mathrm{M}^{+}-\mathrm{C}(7) \mathrm{H}_{2} \mathrm{C}(6) \mathrm{HOH}\right]^{+}$suggests a substituent at $\mathrm{C}-3$ with 85 mass units, such as isovaleryl or 2-methylbutyryl groups (Al-Said et al., 1989). Both, isovaleryl and 2-methylbutyryl groups have been reported as substituents of $D a$ tura alkaloids (Lounasmaa and Tamminen, 1993). On the basis of these MS data the structure of $\mathbf{1 4}$ was tentatively determined as 3-isovaleroyloxy-6hydroxytropane (valeroidine, previously found in Datura sanguinea, Lounasmaa and Tamminen, 1993) or 3-(2'-methylbutyryloxy)-6-hydroxytropane (an alkaloid which has not been found in plants). For unambiguous identification of $\mathbf{1 4}$ however, it must be isolated in pure state.

The alkaloid spectra of the plants grown in Egypt and Bulgaria were different. Thirty-eight alkaloids were found in the roots (21 for Egyptian and 31 for Bulgarian plants). Only 15 of them were found to be common for the roots of Egyptian and Bulgarian plants. Tropine was main alkaloid in the roots of Egyptian plants whereas hyoscyamine was main alkaloid in the Bulgarian ones.

Twenty-one alkaloids (10 for Egyptian and 15 for Bulgarian plants) were detected in the leaves 
Table I. Alkaloids identified in D. innoxia plants cultivated in Egypt (Eg) and Bulgaria (Bg).

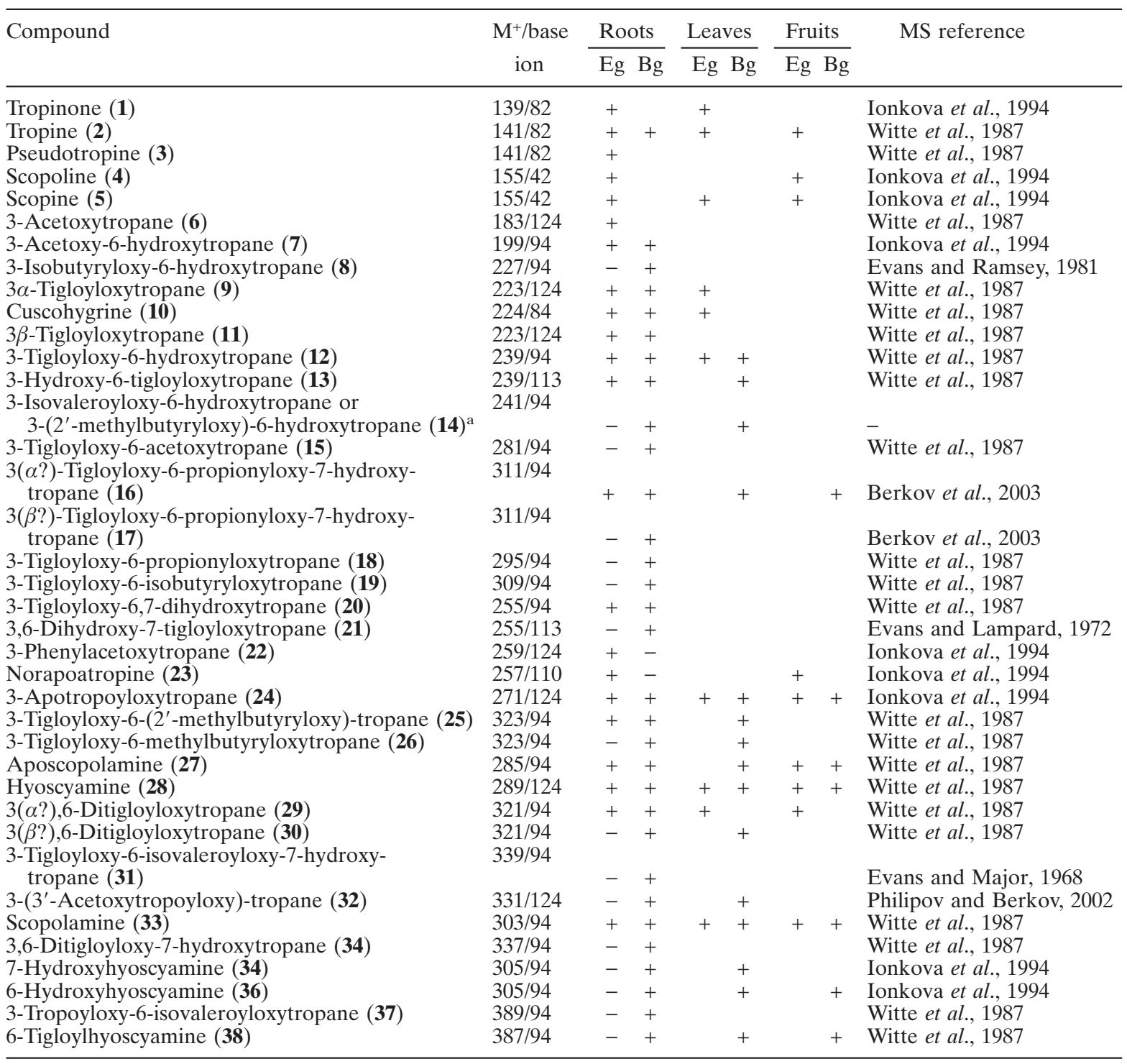

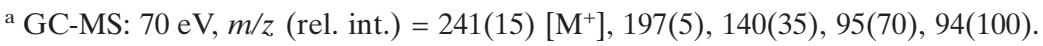

and only 4 of them were found to be common for both origins. Scopine was main alkaloid in the leaves of the Egyptian plants whereas hyoscyamine was main compound in the leaves of Bulgarian plants.

Twelve alkaloids ( 9 for Egyptian and 7 for Bulgarian plants) were found in the fruits, 4 of them were common for both origins. Scopolamine was main compound in the fruit alkaloid fractions of both Egyptian and Bulgarian plants.
As a comparison, Witte et al. (1987) reported 3,6-ditigloyloxytropane and scopolamine as main alkaloids in the roots and leaves, respectively, of D. innoxia plants grown in Germany. Thirty-four alkaloids (32 for the roots and 6 for the leaves) were detected in these plants by GC-MS. The authors reported ten other alkaloids, which we did not detect in our samples. Some of these alkaloids, such as hygrine, were presented in relatively high amounts in the alkaloid fractions. 
Our results as well as the results of Witte et al. (1987) clearly show that the alkaloid spectra and accumulation in $D$. innoxia are strongly influenced by the environmental factors. Concerning this, as well as the relatively high number of alkaloids synthesized by the plant, it is not surprising that new alkaloids are reported for samples from different geographical locations. In such a case, the application of tropane alkaloids for chemotaxonomical studies should be very cautiously. It could be reasonable when the plants are cultivated at identical growth conditions. Thus, the genotype only will determine the alkaloid spectra and accumulation.

\section{Experimental}

Seeds of Datura innoxia Mill. were received from Prof. Dr. El-Shazly (Faculty of Pharmacy, Zagazig University, Egypt) and Dr. Ionkova (Pharmacy Faculty, Sofia Medical University, Bulgaria). Plants were grown at the Botanical Garden of Zagazig University and in the greenhouse of the Institute of Botany, Bulgarian Academy of Sciences (BAS). The samples were collected at florescence.

Al-Said M., Evans W., and Grout R. (1989), Alkaloids of Erythroxylum hypericifolium leaves. Phytochemistry 28, 3211-3215.

Berkov S., Pavlov A., Kovatcheva P., Stanimirova P., and Philipov S. (2003), Alkaloid spectrum in diploid and tetraploid hairy root cultures of Datura stramonium. Z. Naturforsch. 58c, $42-46$.

Griffin W. and Lin D. (2000), Chemotaxonomy and geographical distribution of tropane alkaloids. Phytochemistry 53, 623-637.

Evans W. and Major V. (1968), The tropane alkaloids of genus Datura, section Brugmansia. Part V. Alkaloids of D. sanguinea R., and P., and related esters of tropane-3,6,7-triol. J. Chem. Soc. (C), 2775-2778.

Evans W. and Lampard J. (1972), Alkaloids of Datura suaveolens. Phytochemistry 11, 3293-3298.

Evans W. and Ramsey A. (1981), Tropane alkaloids from Anthocercis and Anthotroche. Phytochemistry 20, 497-499.
Voucher specimens were deposited at the herbarium of Institute of Botany, BAS (SOM-Co 978) and Flora of Egypt 94, 6(5).

Plant samples were prepared and analyzed by GC-MS after the method described in Witte et al. (1987). The identities of the alkaloids were confirmed by comparing the measured mass spectral data with those obtained from the literature and database NIST 98 (a Hewlett Packard Mass Spectral Library, Palo Alto, CA, USA; see Table I for MS references).

\section{Acknowledgements}

This work was partially supported by National Research Foundation of Bulgaria (project MU-SS 1101). The authors are thankful to Ms A. Backhas (Institut für Pharmazie und Molekulare Biotechnologie) and Ms P. Kraemer (Organisch-Chemisches Institut, Universität Heidelberg, Germany) for GC-MS measurements. Furthermore, they gratefully acknowledge Prof. Dr. A. El-Shazly for plant identification.

Ionkova I., Witte L., and Alfermann H.-A. (1994), Spectrum of tropane alkaloids in transformed roots of Datura innoxia and Hyoscyamus $\times$ gyorffyi cultivated in vitro. Planta Med. 60, 382-384

Lounasmaa M. and Tamminen T. (1993), The tropane alkaloids. In: The Alkaloids (Brossi A., ed.), Vol. 44. Academic Press, New York, pp. 1-114.

Philipov S. and Berkov S. (2002), GC-MS investigation of tropane alkaloids in Datura stramonium. Z. Naturforsch. 57c, 559-561.

Witte L., Muler K., and Alfermann H.-A. (1987), Investigation of the alkaloid pattern of Datura innoxia plants by capillary gas-liquid-chromatography-massspectrometry. Planta Med. 53, 192-197. 